\title{
HUBBLE SEQUENCE AS A TEMPORAL EVOLUTION SEQUENCE
}

\author{
XIAOLEI ZHANG \\ Harvard-Smithsonian Center for Astrophysics \\ 60 Garden St., MS 78 \\ Cambridge, MA 02138, USA
}

The results from Hubble Space Telescope's Medium Deep Survey and Deep Fields indicate that there exists far more blue spiral galaxies at the intermediate and high redshifts than at the present epoch. A natural question therefore is: what have become of these excess late type galaxies as the Universe aged?

Recent theoretical work on the secular dynamical evolution of spiral galaxies (Zhang 1996, ApJ, 457, 125; Zhang 1998, ApJ, 494, in press) indicates that an average spiral galaxy undergoes significant morphological evolution during its lifetime, in the process of which its nuclear bulge increases in size through the radial accretion of both stellar and gaseous mass, and its Hubble type changes from late to early and eventually to elliptical galaxies of varying ellipticities. The underlying mechanism responsible for this evolution is a nonlinear and dissipative energy and angular momentum exchange process between the spiral density wave and the basic state of the galactic disk. During this process the angular momentum of the inner disk material gets loaded onto the spiral density wave, which transports this angular momentum outward, and eventually the angular momentum gets unloaded from the wave back to the material in the outer disk. As a result, the disk material in the inner disk spirals inward, and in the outer disk spirals outward, so with time a more and more centrally-concentrated configuration is obtained, together with an extended outer envelope.

Since the rate of spiral-induced secular evolution is proportional to the square of the wave amplitude and to the square of the pitch angle of the spiral, the interactions among galaxies generally accelerate the speed of the morphological evolution of the participating members through the excitation of large-amplitude, open spiral wave patterns. This appears to be the underlying process responsible for the rapid steepening of the galaxy morphology-environment relation with decreasing redshift. 